\title{
Arzneimittelentwicklung zu vernachlässigten Krankheiten
}

\author{
Versuchsfeld für Open-Innovation-Initiativen
}

Katrin Gerlinger, Büro für Technikfolgen-Abschätzung beim Deutschen Bundestag (TAB), Karlsruher Institut für Technologie (KIT),

Neue Schönhauser Straße 10, 10178 Berlin (gerlinger@tab-beim-bundestag.de), (D) orcid.org/0000-0001-9454-2625

Krankheiten, die fast ausschließlich in armen Ländern auftreten, werden seit Jahrzehnten in der medizinischen Forschung und Entwicklung vernachlässigt. Eine Ursache wird in der zahlungsschwachen Nachfrage gesehen. Sie kann die aufwendige kommerzialisierte Produktentwicklung nicht in ausreichendem Maße anreizen. Seit etlichen Jahren werden Wege gesucht, das systemische Problem des patentbasierten Innovationsprozesses zumindest bei armutsassoziierten Krankheiten abzumildern. Einige Akteure testen, inwiefern sich eine Grundidee der Open-Source-Softwareentwicklung - die Zusammenarbeit Freiwilliger bringt Produkte hervor, die keiner Firma allein gehören - auf die Arzneimittelentwicklung übertragen lässt. Dafür öffnen sie unterschiedliche Elemente des pharmakologischen Innovationsprozesses für eine gemeinsame Nutzung und Weiterentwicklung. Sie bezeichnen dies teilweise als Open Innovation. Der Artikel stellt relevante Initiativen vor.

\section{Drug development for neglected diseases}

Field test for various open innovation initiatives

There is a lack of sufficient research and development regarding diseases almost exclusively affection poor countries. One reason is seen in the financially weak demand, which can not sufficiently stimulate the complex commercialized product development. In recent years, national and international measures have been discussed and partly been implemented to attenuate the systemic problem of the patent-based innovation process, at least for poverty-related diseases. Some Stakeholders are testing how the idea of open source software development - the collaboration of volunteers generate products that are not owned by a company - can be transferred to drug development. They open different elements of the pharmacological innovation process for sharing and further drug development. Partly they call this Open Innovation. The article presents relevant initiatives.

KEYWORDS: neglected diseases, research and development, market failure, open innovation

This is an article distributed under the terms of the Creative Commons Attribution License CCBY 4.0 (https://creativecommons.org/licenses/by/4.0/)

https://doi.org/10.14512/tatup.26.1-2.37

Eingereicht: 15. 03.2017. Angenommen: 07.06.2017
Für poverty-related and neglected diseases (PRND) gibt es bisher keine einheitliche Definition. Überwiegend werden die sogenannten Big 3 (HIV/Aids, Tuberkulose, Malaria), 17 von der WHO definierte vernachlässigte Tropenkrankheiten (von Denguefieber über Lepra bis Wurmerkrankungen; WHO 2015, S. $70 \mathrm{ff}$.) sowie einige Atemwegs- und Durchfallerkrankungen als vernachlässigt und armutsassoziiert aufgefasst. Es sind ausnahmslos Infektionskrankheiten (TAB 2017, S. 54). Diese Krankheiten, die fast ausschließlich in armen Ländern auftreten, werden seit Jahrzehnten in der medizinischen Forschung und Produktentwicklung (FuE) vernachlässigt. Verfügbare Medikamente werden oft nicht an die Erfordernisse des globalen Südens angepasst, haben erhebliche Nebenwirkungen und verlieren mit der Zeit ihre Wirksamkeit. Teilweise fehlen wirksame Medikamente ganz, wie die Ebolaepidemie 2014 der Welt auf dramatische Weise zeigte. Diese Situation wird zunehmend als globales Gesundheitsproblem wahrgenommen und Wege zur Verbesserung gesucht. Die WHO hat sowohl makroökonomische als auch gesundheitspolitische Analysen (CMH 2001; CIPIH 2006) in Auftrag gegeben, die Weltgesundheitsversammlung Empfehlungen abgeleitet (u. a. WHA 2008). Einigkeit besteht, dass weder der öffentliche Sektor noch die Privatwirtschaft allein die Kapazitäten besitzen, um Arzneimittel gegen diese Krankheiten zu entwickeln. Unterschiedliche Ansichten gibt es zur generellen Eignung des patentbasierten Innovationssystems und folglich werden die in den letzten Jahren diskutierten und teilweise bereits etablierten Maßnahmen je nach Standpunkt unterschiedlich bewertet und unterstützt. Mitunter wird angemerkt, dass die Vielzahl an Maßnahmen zu Unübersichtlichkeit führt und einen zunehmenden Abstimmungsaufwand erfordert.

Vor diesem Hintergrund hat der Ausschuss für Bildung, Forschung und Technikfolgenabschätzung des Deutschen Bundestags das Büro für Technikfolgen-Abschätzung beim Deutschen Bundestag (TAB) beauftragt, einen Überblick über Maßnahmen zur FuE-Stärkung gegen PRND zu geben. Das TAB hat Initiativen öffentlicher und privater FuE-Akteure sowie politische Maßnahmen zur Förderung des PRND-Engagements untersucht. Es wurde hinterfragt, welche Maßnahmen lediglich diskutiert, welche getestet oder umfangreich angewendet werden. Diese am 
pharmakologischen Innovationsprozess ausgerichtete Darstellung bildet zusammen mit einer vertiefenden Ist-Zustandsanalyse des FuE-Standorts Deutschland die Basis zur Ableitung von Handlungsoptionen für den Deutschen Bundestag (TAB 2017).

Im Rahmen des Projekts wurde deutlich, dass nationale und multilaterale politische Weichenstellungen vorrangig öffentliche FuE-Aktivitäten stärken, die Arzneimittelnachfrage bündeln sowie große Abnahmemengen langfristig sichern und kanalisieren. Diverse FuE-Akteure starteten Initiativen zur Senkung des FuE-Defizits. Einige begannen, Elemente des Innovationsprozesses für eine gemeinsame Nutzung zu öffnen und bezeichneten dies teilweise als Open Innovation. Anhand des TAB-Projekts kann eine Art Praxis-

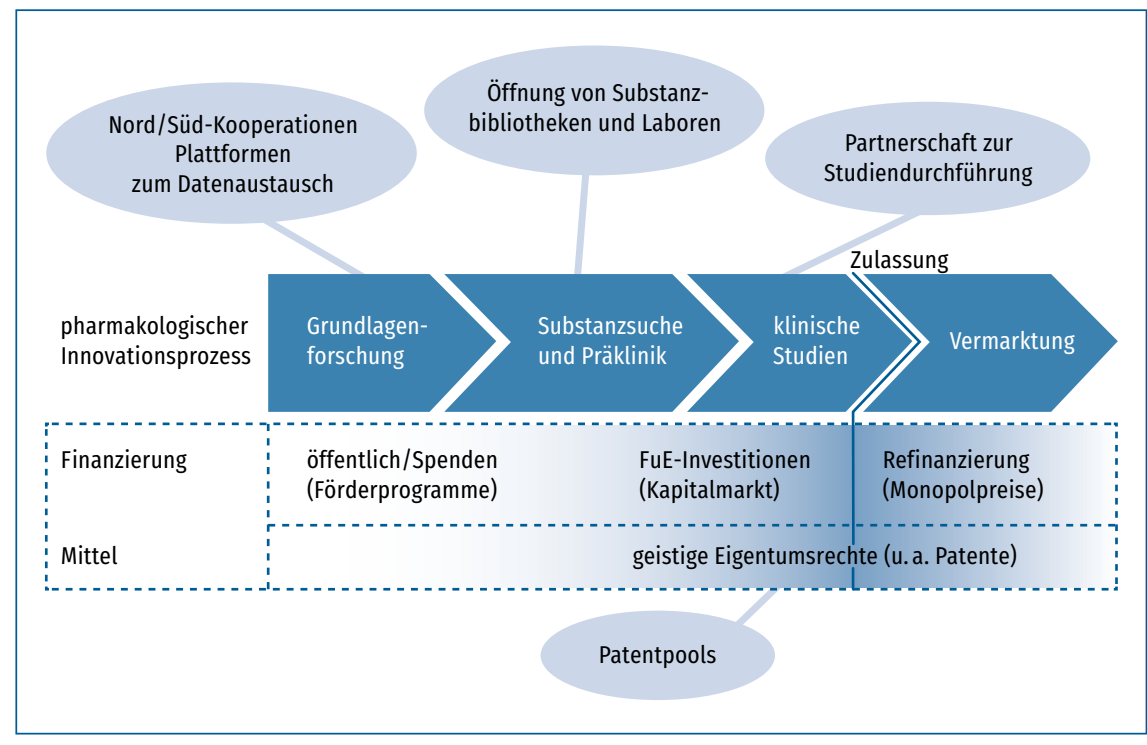

Abb.1: Open-Innovation-Elemente bei der Arzneimittelentwicklung zu vernachlässigten armutsassoziierten Krankheiten. Quelle: Eigene Darstellung bericht zur Öffnung und gemeinsamen Nutzung unterschiedlicher FuE-Elemente gegeben werden. Der TAB-Bericht kann ein Ausgangspunkt sein für eine tiefergreifende wissenschaftliche Befassung mit den Gemeinsamkeiten und Unterschieden anderer Ansätze zu Open Source, Open Science oder Open Innovation (TAB 2017). Solch eine Befassung lag jedoch nicht im Projektauftrag des TAB.

Der nachfolgend skizzierte pharmakologische Innovationsprozess mit seinen Entwicklungsphasen und Finanzierungsmechanismen dient als Ordnungsrahmen für die Darstellung von Ansätzen zur Öffnung und gemeinsamen Nutzung von FuE-Elementen und -infrastrukturen, die innerhalb des TAB-Projektes sichtbar wurden. Die Darstellung erhebt keinesfalls den Anspruch auf Vollständigkeit, dafür ist der Beobachtungsraum zu groß.

\section{Der pharmakologische Innovationsprozess: Ansatzpunkte für Open Innovation}

Arzneimittel unterliegen in den Industrieländern einem Verbotsprinzip mit Erlaubnisvorbehalt. Die Produktionserlaubnis ( $\mathrm{Zu}$ lassung) wird nur erteilt, wenn für neue Mittel deren Sicherheit und Wirksamkeit belegt sowie eine qualitätsgesicherte Produktion und ein Sicherheitsmonitoring vom Hersteller garantiert werden. Dafür sind vielfältige FuE-Aktivitäten nötig. Der Ablauf aufeinander aufbauender Produktentwicklungsphasen wird als pharmakologischer Innovationsprozess bezeichnet (Abb. 1). Jenseits der Grundlagenforschung läuft er in vielen Ländern weitgehend in kommerziellen Strukturen ab. Dabei werden FuE-Investitionen über geistige Eigentumsrechte (Patente, Unterlagenschutz, Datenexklusivität; TAB 2017, S. 170 ff.) staatlich geschützt, sodass der Rechteinhaber für einen begrenzten Zeitraum über die gewerbliche Nutzung allein entscheiden darf.

Diese Rechte können mittels Lizenzen übertragen und damit verwertet werden. Sie sind ein wichtiger Garant, mit dem Kapital für die Produktentwicklung akquiriert werden kann. Nach der Zulassung ermöglichen sie befristete Monopolpreise, bei denen die FuE-Aufwendungen ein Preisbestandteil sind, die jedoch auch positive Investmentreturns ermöglichen. Die hohen Monopolpreise neuer Medikamente sind nahezu für jeden Patienten, der diese benötigt, eine große Herausforderung. In den Industrieländern wird diese erhebliche finanzielle Belastung des Einzelnen solidarisch finanziert. Bei vielen in den Industrieländern verbreiteten Krankheiten funktioniert dieser Marktmechanismus im Großen und Ganzen, was jedoch keinesfalls heißt, dass es keine Kritik gibt.

Bei schwacher Nachfrage - wenn ein neues Medikament nur selten benötigt wird und/oder wenn Betroffenengruppen u. a. wegen fehlender sozialer Sicherungssysteme finanzschwach sind - versagt dieser Mechanismus. Denn auch Monopolpreise können nicht beliebig verändert werden und folglich kann keine vollständige Refinanzierung der FuE-Investitionen erwartet werden. Investitionen bleiben aus, forschungsseitige Vernachlässigung folgt. Bei Krankheiten, die vor allem in armen Ländern auftreten, passiert dies seit Jahrzehnten.

Da Marktmechanismen bei PRND nicht funktionieren, wächst bei einigen FuE-Akteuren die Bereitschaft, andere Geschäftsmodelle zu testen. In dieser Konstellation ist die PRNDFuE zu einem Versuchsfeld für unterschiedliche Open-Innovation-Initiativen geworden.

Diese Initiativen sind Praxisexperimente von engagierten Freiwilligen, die sich zum Ziel setzen, das bestehende FuE-Defizit zu senken, und die damit einen Beitrag zur Verbesserung der globalen gesundheitlichen Situation leisten wollen. Im Rahmen des TAB-Projektes fiel auf, dass diese Pragmatiker den ihrerseits genutzten Open-Innovation-Begriff weder aus der wis- 
senschaftlichen Literatur ableiteten (z. B. Bianchi et al. 2011; Chesbrough 2003; Chesbrough und Bogers 2014) noch eine dezidierte Auseinandersetzung um die Charakteristik, die Einordnung oder genaue Abgrenzung führten. Einer der Aktivisten will mit seinen Initiativen testen, inwiefern sich das Geschäftsmodell der Open-Source-Softwareentwicklung auf die Arzneimittelentwicklung übertragen lässt, d.h. die Quellen (in diesem Fall Substanzen, Daten, Ergebnisse) werden offengelegt und die Zusammenarbeit Freiwilliger bringt Produkte hervor, die keiner Firma alleinig gehören (Guth 2010). Von Open Innovation wird wohl auch deshalb gesprochen, weil die eigentliche Herausforderung darin besteht, jenseits der Grundlagenforschung auch den aufwendigen und verantwortungsvollen Prozess der Produktentwicklung in den Blick zu nehmen, um die benötigten Arzneimittel auch bis zur Zulassung zu bringen.

\section{Grundlagenforschung}

Die Grundlagenforschung wird bisher überwiegend in öffentlichen Forschungseinrichtungen durchgeführt. Sie dient dem besseren Verständnis einer Krankheit. U. a. werden krankheitsspezifische Angriffspunkte oder Biomarker gesucht (sog. Targets). Dafür werden biologische Materialien benötigt, die nur in krankheitsendemischen Gebieten genommen werden können. In den Industrieländern wird solches Material zunehmend in Biobanken zusammengeführt und gelagert sowie mit modernster Labortechnik (u. a. DNA-Sequenzierungsautomaten, höchstauflösende Mikroskopiertechnik) standardisiert oder gar automatisiert aufbereitet und analysiert. In den Industrieländern wurden spezialisierte Forschungszentren aufgebaut, die Technik teilweise zu sogenannten Forschungsinfrastrukturen zusammengeführt (z. B. European Molecular Biology Laboratory). Die Zentren definieren mit ihren Förderern Forschungsschwerpunkte und -programme. Einige nehmen nicht nur national oder europäisch bedeutsame medizinische Herausforderungen, sondern auch PRND in ihre Forschungsagenda auf. Im Rahmen des TAB-Projektes wurden die vom britischen Centre for Genomics and Global Health initiierten Aktivitäten zur Bekämpfung von Infektionskrankheiten, allen voran Malaria, als beispielgebend eingeschätzt, das bestehende Forschungsdefizit zu senken. Folgende internationale Forschungsnetzwerke und Datenaustauschplattformen sind dadurch entstanden:

- Das Malaria Genomic Epidemiology Network (MalariaGEN) will einen digitalen Atlas der Genomvarianz der relevantesten Malariaerreger erstellen. Am Netzwerk beteiligen sich inzwischen knapp 200 Forscher in über 30 Ländern. Arbeitsschwerpunkte sind Genomsequenzierungen, sowie die digitale Aufbereitung und Bereitstellung der genetischen Daten und der daraus gewonnenen Analyseergebnisse. Der Atlas ist für alle Netzwerkteilnehmer frei zugänglich.

- Das Plasmodium Diversity Network Africa will die genetische Plasmodiendiversität und die Resistenzentstehung in
Subsahara-Afrika erforschen und zeitnah über aufkommende Resistenzen informieren. Beteiligte afrikanische Institute sammeln kontinuierlich Blutproben von Malariaerkrankten und schicken sie zum Wellcome Trust Sanger Institute nach Großbritannien. Dort werden die Plasmodiengenome sequenziert und Daten und Analyseergebnisse über den Atlas der Genomvarianz für das Netzwerk bereitgestellt.

Für den Atlas als zentrale Datenaustauschplattform wurden ethische Regeln und Datenschutzstandards definiert. Sie sollen sowohl die Souveränität der Probenlieferanten als auch den verantwortlichen Zugang zu den genetischen Daten und deren Nutzung sichern (MalariaGEN 2008). Die Lieferanten behalten die Verantwortung über das bereitgestellte Material und die genetischen Daten, sie müssen in deren netzwerkinterne Nutzung einwilligen. Ergebnisse aus den Netzwerkprojekten werden veröffentlicht und über den Atlas netzwerkintern zugänglich gemacht, es sei denn, die Projektleitung visiert eine Patentierung an. Letzterer werden enge Grenzen gesetzt. Ein Patent soll nur als Anreiz zur weiteren Produktentwicklung dienen (MalariaGEN 2008, S. 3). Falls Lizenzgebühren anfallen, sollen diese in die teilnehmenden südlichen Gemeinschaften fließen.

\section{Substanzscreening und Präklinik}

Je detaillierter Wissen aus der Grundlagenforschung ist, desto zielgerichteter kann nach Molekülen gesucht werden, die an krankheitsspezifische Targets binden (Schlüssel-Schloss-Prinzip). Ähnlich wie biologische Proben in Biobanken werden potenziell bindungsfähige Moleküle in Substanzbibliotheken gesammelt und aufbewahrt. Diese Moleküle können Ausgangsstoffe für Arzneimittel sein und sind patentierbar. Pharmaunternehmen haben umfangreiche Substanzbibliotheken aufgebaut, deren Zugang im Regelfall streng limitiert ist. Einige Firmen haben begonnen, ihre Substanzbibliotheken für PRND-Aktivitäten ein Stück weit zu öffnen. Sie scannten Millionen Substanzen auf ihre Bindungsfähigkeit an identifizierte PRND-Targets entweder allein oder mit ausgewählten gemeinnützigen Product Development Partnerships (PDP). Die diesbezüglich weitreichendste Initiative startete der Pharmakonzern GlaxoSmithKline (GSK). Firmenintern wurden aus ca. 2 Millionen Substanzen 13500 Moleküle herausgefiltert, die an malariaspezifische Targets binden.

Das Screening ist jedoch nur der erste Schritt in der präklinischen Produktentwicklung. Weitere Analysen sind notwendig, um aus der großen Zahl bindungsfähiger Moleküle die besten Leitstrukturen herauszufiltern und nur diejenigen mit der wahrscheinlich besten Verträglichkeit und größten Wirksamkeit zu Substanzkandidaten weiterzuentwickeln. Das geschieht erst anhand unterschiedlicher Labortests, dann mit Tierversuchen (Präklinik).

Parallel zum Substanzscreening etablierte GSK einen Pool for Open Innovation against neglected tropical diseases, speiste 
die Daten und geistigen Eigentumsrechte zu den Molekülen ein und erlaubte die Nutzung für FuE-Aktivitäten zu weiteren 21 PRND (Boseley 2010). Dieser zunächst firmeninterne Pool wurde Ende 2011 zum globalen Patentpool WIPO Re:Search ausgeweitet (s. u.). Ein weiteres Element der Open-InnovationInitiative war die Einrichtung eines Open-Lab am GSK-Forschungscampus Tres Cantos (Spanien), das von der firmeneigenen Stiftung grundfinanziert wird. Dort können auch externe Forscher an der Produktentwicklung gegen PRND arbeiten, sofern sie Daten, Ergebnisse und möglicherweise resultierende geistige Eigentumsrechte in den Patentpool einspeisen.

Das europäische Marie-Skłodowska-Curie-Programm zur personellen Forschungsförderung ist 2015 für diesbezügliche Aktivitäten geöffnet worden. Es finanziert auch mehrjährige PRND-Forschungstätigkeiten am Open-Lab. Für Wissenschaftler, die sich an ihren Heimatinstituten an den Forschungsaktivitäten beteiligen möchten, wurde eine sogenannte MalariaBox in Kooperation mit Novartis und einer gemeinnützigen PDP entwickelt. Die Box enthält 400 potenziell bindungsfähige Moleküle und wird Wissenschaftlern kostenlos bereitgestellt, wenn sie resultierende Daten und Ergebnisse frei zugänglich machen.

Die PDP Drugs for Neglected Diseases Initiative assoziiert mit dem Begriff Open Innovation ähnliche Elemente wie GSK. Auch sie nennt die Öffnung von Substanzbibliotheken sowie den Zugang und die Nutzung von geistigen Eigentumsrechten, Daten und Wissen für FuE-Aktivitäten zu PRND (DNDi 2011).

\section{Patentpools}

Erste Ideen, Patente zur PRND-Bekämpfung gemeinsam zu nutzen, entstanden nach der Jahrtausendwende. Die Weltgesundheitsversammlung bewertete dies als machbares Instrument zur Verbesserung der Arzneimittelverfügbarkeit in Entwicklungsländern (Eppinger 2014, S. 195). Die Idee wurde weiterentwickelt und wird seit einigen Jahren in zwei Varianten getestet. Eine Variante soll den möglichst kostengünstigen Zugang zu bereits zugelassenen Arzneimitteln für Entwicklungsländer ermöglichen, sogenannte downstream pools (z. B. der Medicine Patent Pool). Für FuE sind upstream pools relevant. Durch sie werden definierte FuE-Aktivitäten erlaubt. Für PRND-FuE ist der bei der World Intellectual Property Organization geführte und als OpenInnovation-Plattform bezeichnete Patentpool WIPO Re:Search von besonderer Bedeutung. Er ist aus der GSK-Initiative hervorgegangen. Patenthalter weltweit sind aufgerufen, ihre Daten und Informationen zu ihren patentierten Substanzen und Verfahren in den Pool einzuspeisen und die kostenlose Nutzung zur Arzneimittelentwicklung gegen ausgewählte PRND zu erlauben. Alle Teilnehmer verpflichten sich, im Falle einer Produktzulassung sozialverträgliche Produktionslizenzen zu vergeben (die Höhe der Lizenz wird in einem transparenten Verfahren anhand des Bruttoinlandsproduktes festgelegt, die ärmsten Länder erhalten diese Lizenz kostenlos).

\section{Klinische Studien}

Wenn für Substanzkandidaten grundlegende Fragen vor allem zur Toxizität geklärt wurden und ein therapeutischer Nutzen erwartet wird, kann mit Prüfungen am Menschen begonnen werden. Dafür gibt es nahezu weltweit gültige Standards. In den Industrieländern sind spezifische Regularien für die Durchführung klinischer Studien (Arzneimittelgesetz in Deutschland) vorhanden. Klinische Studien sind grundsätzlich genehmigungspflichtig. Studienverantwortlichkeit und Haftung bei möglichen Schäden müssen definiert und abgesichert werden. Bei PRND müssen die klinischen Studien zum Wirksamkeitsnachweis in den jeweils endemischen Ländern durchgeführt werden. Dort müssen oftmals Regularien und die notwendigen medizinischen Zentren noch ausgebaut werden. Diese FuE-Etappe ist in der Regel sehr kostenintensiv.

Im TAB-Projekt fiel auf, dass in der Phase klinischer Studien Begriffsbildungen mit „Open“ kaum verwendet werden. Obwohl in klinischen Studien vielfältige medizinisch hochrelevante Daten erhoben werden, ist ein Zugang nicht nur wegen möglicher geistiger Eigentumsrechte, sondern auch wegen der Personenund Gesundheitsbezüge der Daten, verbunden mit ärztlichen Schweigepflichten, bisher problematisch. Die Finanzierung dieser FuE-Phase bis zur Marktzulassung ist eine große Herausforderung, weil einerseits privates Kapitel kaum in Frage kommt und andererseits das Know-how insbesondere zur Produktzulassung vor allem in der Industrie vorhanden ist. Seit Jahren werden für PRND-FuE alternative Finanzierungsmechanismen diskutiert. In der WHO wird seit etlichen Jahren über globale Finanzierungsfonds gesprochen, ohne dass sich eine Akzeptanz vor allem bei den potenziellen Geberländern abzeichnet. Diese Länder setzen ihre öffentlichen Mittel lieber im Rahmen nationaler und europäischer Programme und Förderstrukturen ein (TAB 2017, S. 270 ff.). Dazu kommen Spenden von einigen großen Stiftungen, allen voran die Gates-Stiftung und der WellcomeTrust (ausführlich in Moran et al. 2017).

Ein Ansatz der gemeinschaftlichen Finanzierung und Durchführung klinischer Studien zu PRND ist die 2003 gestartete European and Developing Countries Clinical Trials Partnership (EDCTP). Sie wird weitgehend durch die öffentlichen Haushalte der Europäischen Kommission und der teilnehmenden europäischen Länder gespeist, um gemeinsam mit teilnehmenden afrikanischen Ländern klinische Studien vor Ort durchzuführen. Zwar werden satzungsgemäß Daten, Ergebnisse und möglicherweise bestehende geistige Eigentumsrechte innerhalb der Partnerschaft gemeinsam genutzt, der Begriff Open Innovation wird von der EDCTP jedoch nicht mit den Aktivitäten assoziiert.

\section{Engagement deutscher FuE-Akteure}

Im Rahmen des TAB-Projekts wurde deutlich, dass der Forschungsstandort Deutschland über eine breite Basis hochkompetenter öffentlicher und privatwirtschaftlicher FuE-Einrichtungen 
verfügt, die sich in Bezug auf PRND vor allem in klassischen, patentbasierten Innovationsstrukturen engagieren. Der offene Zugang zu wissenschaftlichen Publikationen von Forschungsergebnissen (Open Access) aus der Grundlagenforschung setzt sich mehr und mehr durch, da die Förderrichtlinien der öffentlichen Forschungsprogramme dies zunehmend verankern. Bei den vorgestellten, als Open Innovation gelabelten Initiativen (z. B. MalariaGEN, Screening-Initiativen, Open-Lab, Patentpools) ist das Engagement deutscher FuE-Akteure vor allem im Vergleich zu britischen Akteuren bisher höchst verhalten. Weder gingen von deutschen FuE-Akteuren relevante Open-Innovation-Initiativen aus, noch beteiligen sie sich an bereits gestarteten Initiativen in nennenswertem Umfang. Die EDCTP-Beteiligung kann jedoch als Indiz gewertet werden, dass sie die gemeinsame Nutzung von Daten, Ergebnissen und Nutzungsrechten in einer vereinbarten Partnerschaft nicht per se ablehnen.

\section{Fazit}

Um dem globalen Gesundheitsproblem der unzureichenden Produktentwicklung bei vernachlässigten armutsassoziierten Krankheiten zu begegnen, beginnen sowohl einzelne öffentliche und gemeinnützige Einrichtungen als auch Pharmaunternehmen unterschiedliche Elemente des pharmakologischen Innovationsprozesses zu öffnen. Sie bezeichnen dies teilweise als Beitrag oder Experiment zu Open Innovation, ohne den Begriff genau zu definieren oder sich mit entsprechenden theoretischen Konzepten dezidiert auseinanderzusetzen. Durch das TAB-Projekt kann lediglich ein Praxisbericht unterschiedlicher Initiativen geliefert werden. Bei dieser Praxisbeobachtung wurde deutlich, dass sich jenseits des völlig freien Zugangs zu publizierten Forschungsergebnissen (Open Access) derzeit keinesfalls ein
Die beispielhaft vorgestellten Open-Innovation-Initiativen gelten als Experimente und sind nicht unumstritten. Befürworter sehen darin einen möglichen potenziellen Wegbereiter für neue Ansätze in der Pharmaforschung, die zunehmend demonstrieren, dass offene Ansätze Innovationen befördern. Sie weisen jedoch darauf hin, dass es zu früh ist, um deren Effekte und Wirkungen zu bewerten (DNDi 2011). Kritiker bezweifeln, dass privatwirtschaftliche FuE-Akteure sich in ausreichendem Maße beteiligen und die notwendigen finanziellen Mittel zur effizienten und notwendigen Produktentwicklung bereitgestellt werden können (Moldenhauer et al. 2012, S. 128 f.). Insbesondere die Finanzierung der aufwendigen letzten Phasen der Produktentwicklung und die Frage, wer für klinische Studien oft mit tausenden Patienten in offenen Strukturen die Gesamtverantwortung und Haftung übernimmt, gilt es zu klären. Bisher gibt es keine Belege, dass mit diesen Ansätzen dringend benötigte Arzneimittel effizient bis zur Zulassung gebracht werden können, was jedoch auch daran liegt, dass die Entwicklungszyklen neuer pharmakologischer Wirkstoffe bis zur Marktzulassung sehr lang sowie Aufwands- und Kostenkomponenten bei jeder Produktentwicklung sehr spezifisch sind und kaum verallgemeinert werden können.

Trotz der noch fehlenden Wirksamkeitsbeweise können diese Initiativen Möglichkeiten und Grenzen aufzeigen sowie Anregungen liefern für andere Bereiche mit versagenden Marktmechanismen (z. B. seltene Erkrankungen oder zunehmende Antibiotikaresistenzen). Antibiotikaresistenzen werden erst seit wenigen Jahren als globales Problem wahrgenommen und die Auseinandersetzung, mit welchen Maßnahmen Forschung und Produktentwicklung befördert und finanziert werden können, beginnt gerade.

Eine vertiefende Analyse der unterschiedlichen Open-Innovation-Initiativen wäre hilfreich, um deren Folgedimensionen besser abschätzen zu können. Eine Debatte zu den dabei

\section{Die Herausforderung bei Open-Innovation-Initiativen im Pharmabereich besteht darin, den gesamten Innovationsprozess bis zur Produktzulassung in den Blick} zu nehmen und abzusichern.

gänzlich freier Zugang zu Daten, Infrastrukturen oder geistigen Eigentumsrechten abzeichnet. Nur bestimmten FuE-Akteuren werden bei Aktivitäten zu definierten PRND spezifische Zugangs- und Nutzungsmöglichkeiten eröffnet. Open-InnovationElemente werden derzeit nur in Bereichen getestet, in denen Marktmechanismen ohnehin versagen. Dennoch eröffnen die Netzwerke und Partnerschaften Wissenschaftlern weltweit eine Möglichkeit sich an PRND-Projekten zu beteiligen, auch wenn sie selbst keinen direkten Zugang zu modernsten Forschungsinfrastrukturen haben. unterstellten Hoffnungen und Bedenken bietet sich an. Vielleicht könnte die diesbezügliche Zurückhaltung deutscher Akteure überwunden werden. Sie könnten sich vom Engagement z. B. britischer Open-Innovation-Aktivisten im Bereich armutsassoziierter Krankheiten inspirieren lassen. Ein deutlicheres forschungspolitisches Engagement zur Stärkung von Forschung und Produktentwicklung zu vernachlässigten armutsassoziierten Krankheiten im Allgemeinen und zu den Chancen und Risiken von Open Innovation im Besonderen wäre begrüßenswert. 


\section{Literatur}

Bianchi, Mattia; Cavaliere, Alberto; Chiaroni, Davide; Frattini, Frederico; Chiesa, Vittorio (2011): Organisational Modes for Open Innovation in the Bio-Pharmaceutical Industry: An Exploratory Analysis. In: Technovation 31 (1), S. 22-33.

Boseley, Sarah (2010): How GlaxoSmithKline Chief has Changed the Agenda for Big Pharma. Online verfügbar unter https://www.theguardian.com/ business/2010/jan/20/glaxosmithkline-andrew-witty-drugs-developingworld, zuletzt geprüft am 01.06.2017.

Chesbrough, Henry William (2003): Open Innovation: The New Imperative for Creating and Profiting from Technology. Boston: Harvard Business School Press.

Chesbrough, Henry William; Bogers, Marcel (2014): Explicating Open Inovation: Clarifying an Emerging Paradigm for Understanding Innovation. In: Henry William Chesbrough, Wim Vanhaverbeke, Joel West (Hg.): New Frontiers in Open Innovation. Oxford: Oxford University Press, S. 3-28.

CIPIH - Commission on Intellectual Property Rights, Innovation and Public Health (2006): Public Health - Innovation and Intellectual Property Rights. Online verfügbar unter http://www.who.int/intellectualproperty/documents/ thereport/ENPublicHealthReport.pdf?ua=, zuletzt geprüft am 01.06.2017.

CMH - Commission on Macroeconomics and Health (2001): Macroeconomics and Health: Investing in Health for Economic Development. Genf: World Health Organization.

DNDi - Drugs for Neglected Diseases initiative (2011): Striving for Increasingly Open Innovation Models. Online verfügbar unter https://www.dndi.org/ wp-content/uploads/2011/11/Striving_for_increasingly_open_innovation_ models.pdf, zuletzt geprüft am 01.06.2017.

Eppinger, Elisabeth (2014): Patentpools. Eigenschaften, Motive und Implikationen. Wiesbaden: Springer Gabler.

Guth, Robert A. (2010): Glaxo Tries a Linux Approach. Drug Maker Shares its Research Data Online in Test of Open-Source Principles. In: The Wall Street Journal vom 26. 03.2010.

MalariagEN (2008): Joint Policy on Data Sharing, Intellectual Property and Publications. Online verfügbar unter https://www.malariagen.net/sites/default/
files/content/project/files/JointPolicy-DataSharing-IntellectualProperty-

Publications.pdf, zuletzt geprüft am 01.06.2017.

Moldenhauer, Oliver; Frisch, Philipp; Gombe-Götz, Spring (2012): „From Bench to Bedside“: Innovations- und Produktionsprozesse von Medizintools - alternative Konzepte zur Förderung von kommerzieller und nichtkommerzieller FuE, die einen breiten Zugang zu Innovationen sicherstellen. Gutachten im Auftrag des Deutschen Bundestags. Berlin: unveröffentlichtes Manuskript. Moran, Mary et al. (2017): G-Finder 2015. Neglected Disease Research and Development: The Ebola Effect. Online verfügbar unter http://www.policycures.org/ downloads/Y8\%20GFINDER\%20full\%20report\%20web.pdf, zuletzt geprüft am 01. 06. 2017.

TAB - Büro für Technikfolgen-Abschätzung beim Deutschen Bundestag (2017): Neue Arzneimittel gegen vernachlässigte Krankheiten. TAB-Arbeitsbericht Nr. 170, Autorin: Katrin Gerlinger, Berlin: TAB.

WHA - World Health Assembly (2008): Global Strategy and Plan of Action on Public Health, Innovation and Intellectual Property. Online verfügbar unter http://apps.who.int/gb/ebwha/pdf_files/WHA61-REC1/A61_REC1-en.pdf, zuletzt geprüft am 01. 06.2017.

WHO - World Health Organisation (2015): Investing to Overcome the Global Impact of Neglected Tropical Diseases. Third WHO Report on Neglected Tropical Diseases. Online verfügbar unter http://apps.who.int/iris/bitstream/ 10665/152781/1/9789241564861_eng.pdf, zuletzt geprüft am 01. 01.2017.

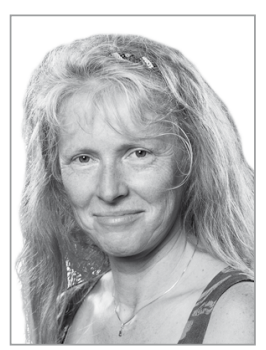

\section{DR. KATRIN GERLINGER}

promovierte in Volkswirtschaftslehre und Statistik am Potsdam-Institut für Klimafolgenforschung. Seit 2006 ist sie Mitarbeiterin im Büro für Technikfolgen-Abschätzung beim Deutschen Bundestag (TAB) mit den Arbeitsschwerpunkten Gesundheitswirtschaft, Innovationssysteme, Entwicklungszusammenarbeit und Datenanalyse.

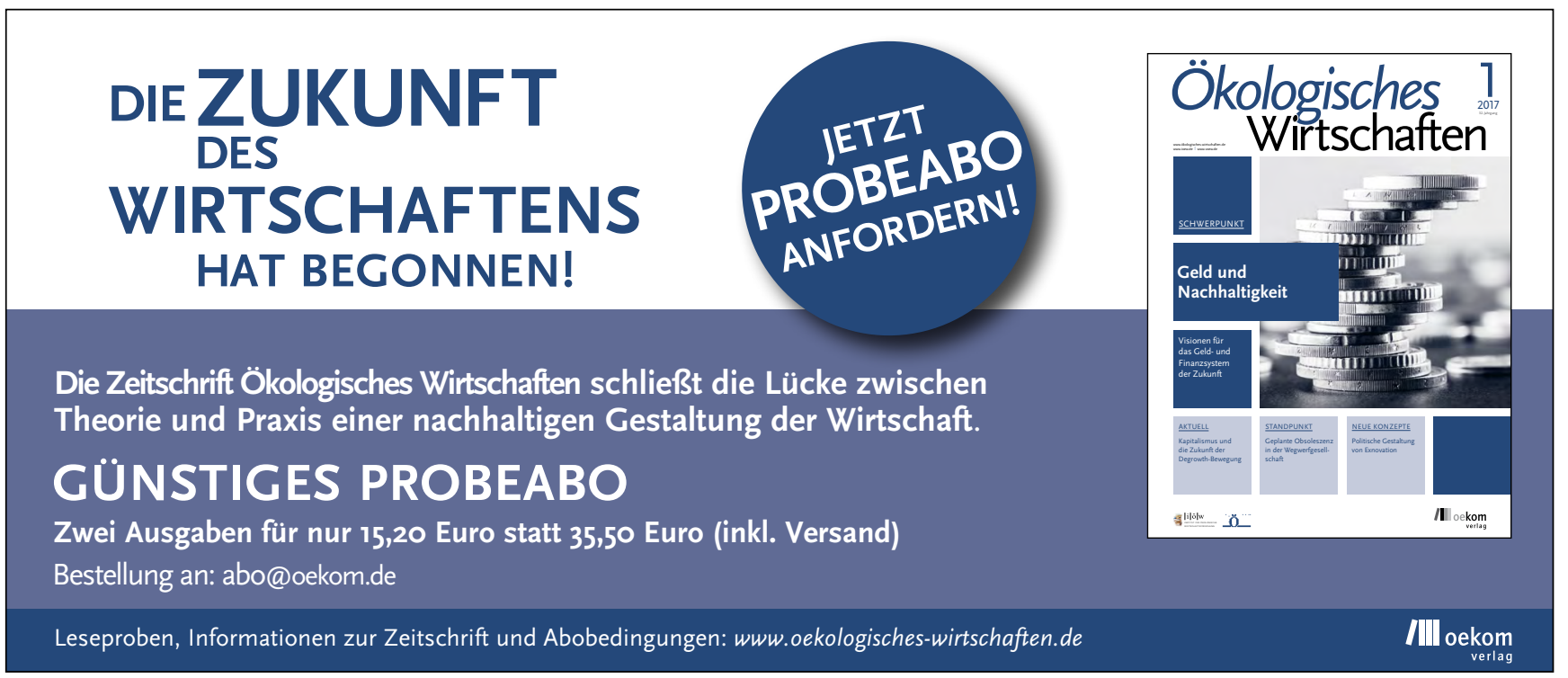

